\title{
Efficacy and Safety of Semi-rigid Ureteroscopic Lithotripsy (URS) for Proximal Ureteral Stone $\geq 10 \mathrm{~mm}$
}

\author{
Muhibullah Bangash, Syed Muhammad Nazim, Salman Jamil, Muhammad Owais Abdul Ghani and Suniya \\ Naeem \\ Department of Urology, The Aga Khan University Hospital, Karachi, Pakistan
}

\begin{abstract}
Objective: To evaluate the efficacy and safety of the semi-rigid URS with pneumatic lithotripsy for the treatment of large ( $\geq 10$ $\mathrm{mm}$ ) proximal ureteral calculi.

Study Design: Cohort study.

Place and Duration of Study: Section of Urology, Department of Surgery, The Aga Khan University Hospital, Karachi, Pakistan, from January 2010 to December 2018.

Methodology: All patients with single, radio-opaque unilateral proximal ureteral stones $\geq 10 \mathrm{~mm}$ were included in the study. Patients with urosepsis, pregnancy, solitary kidney, and indwelling JJ stent or prior percutaneous nephrostomy drainage were excluded. Patients without any visualisation of stone on abdominal X-Ray done at 4 weeks of the procedure were labelled as stone-free.

Results: One hundred and three cases with stones $\geq 10 \mathrm{~mm}$ and fulfilling the selection criteria were included. The mean age was $40.83 \pm 14.92$ years. The mean stones size was $13.33 \pm 4.64 \mathrm{~mm}$. The mean procedure time was $41.50 \pm 15.60$ minutes. Indwelling JJ stents were placed in $49(47.6 \%)$ cases. The stone-free rate was $83.5 \%$ at four weeks with calculated efficiency quotient (EQ) of 0.72 , using a standard equation. The overall complication rate was $27 \%$ with the majority being minor (Clavian-Dindo grade 1). Only one patient had urosepsis (MCG IV). Ancillary procedures were performed in 17 (16.5\%) cases, most commonly the extracorporeal shock wave lithotripsy (ESWL) in 14 (13.5\%) cases, followed by the secondary URS in $3(2.9 \%)$ cases. Conclusion: Semi-rigid URS is an effective and safe procedure for the large proximal ureteral stones, with limited access to flexible instruments.
\end{abstract}

Key Words: Ureteroscopy, Semi-rigid, Stone, Proximal, Clearance, Safety.

How to cite this article: Bangash M, Nazim SM, Jamil S, Ghani MOA, Naeem S. Efficacy and Safety of Semi-rigid Ureteroscopic Lithotripsy (URS) for Proximal Ureteral Stone $\geq 10$ mm. J Coll Physicians Surg Pak 2020; 30(10):1058-1062.

\section{INTRODUCTION}

Ureteral stone disease has a high prevalence and causes significant pain on clinical presentation requiring clinic or emergency department visits. ${ }^{1,2}$ The aim of initial treatment is a relief of pain followed by medical expulsive therapy (MET). Spontaneous passage of larger ureteral stones is usually not facilitated by MET, so this option is generally not considered. ${ }^{3}$

Various modalities available for proximal ureteral stone management are the shock wave lithotripsy (SWL), semi-rigid ureteroscopy (URS), or flexible URS, antegrade percutaneous nephrolithotomy (PCNL) and occasionally laparoscopic or open ureterolithotomy. ${ }^{4}$

Correspondence to: Dr. Syed Muhammad Nazim, Department of Urology, The Aga Khan University Hospital,

Stadium Road, Karachi, Pakistan

E-mail: muhammad.nazim@aku.edu

Received: April 06, 2020; Revised: May 28, 2020;

Accepted: June 13, 2020

DOI: https://doi.org/10.29271/jcpsp.2020.10.1058
Although SWL is the recommended option for managing proximal ureteral calculi, guidelines are less clear for the larger $(>10 \mathrm{~mm}$ ) proximal ureteral stones. Whether SWL or URS should be the first-line management option for patients with largerproximal ureteral stones $(>10 \mathrm{~mm}$ ) is an ongoing controversy. ${ }^{4}$

Recently, with the introduction of the flexible URS and Holmium: yttrium-aluminum-garnet (YAG) laser, this modality is now considered as the initial and preferred treatment option for the proximal ureteral stone. ${ }^{5}$ However, due to very high initial and maintenance costs, the fragility of instruments and accessories, and lack of availability in most institutions in Pakistan, this option is not widely applicable. In developing countries with limited resources like ours, semi-rigid URS can be considered as the initial management option for the large proximal ureteral stones. ${ }^{6}$

The aim of this study was to evaluate the safety and efficacy of semi-rigid URS with pneumatic lithotripsy for proximal ureteral stones $\geq 10 \mathrm{~mm}$ in diameter. 
Table I: Comparison of demographic and clinical factors associated with stone clearance.

\begin{tabular}{|c|c|c|c|}
\hline Variables & Stone free at 4 weeks $(n=86)$ & Not stone free at 4 weeks $(n=17)$ & p-value \\
\hline Mean age & $41.33 \pm 15.36$ & $38.29 \pm 13.04$ & $0.5 *$ \\
\hline Mean Serum Creatinine & $1.35 \pm 1.13$ & $1.86 \pm 1.84$ & $0.14 *$ \\
\hline Mean Stone size & $12.82 \pm 4.31$ & $15.91 \pm 5.53$ & $0.01 *$ \\
\hline Mean Operative time & $41.66 \pm 16.81$ & $40.94 \pm 8.31$ & $0.86^{*}$ \\
\hline $\begin{array}{l}\text { Gender } n(\%) \\
\text { Male } \\
\text { Female }\end{array}$ & $\begin{array}{l}57(66.3) \\
29(33.7)\end{array}$ & $\begin{array}{c}13(76.5) \\
4(23.5)\end{array}$ & $0.41 * *$ \\
\hline $\begin{array}{l}\text { Side } n(\%) \\
\text { Right } \\
\text { Left }\end{array}$ & $\begin{array}{l}42(48.8) \\
44(51.2)\end{array}$ & $\begin{array}{c}5(29.4) \\
12(70.6)\end{array}$ & $0.14^{* *}$ \\
\hline $\begin{array}{l}\text { Stone former } \mathrm{n}(\%) \\
\text { Primary } \\
\text { Recurrent }\end{array}$ & $\begin{array}{l}50(58.1) \\
36(41.9)\end{array}$ & $\begin{array}{c}11(64.7) \\
6(35.3)\end{array}$ & $0.61 * *$ \\
\hline J stenting n (\%) & $39(45.3)$ & $10(58.8)$ & $0.31^{* *}$ \\
\hline Comorbid conditions present & $31(36)$ & $5(29.4)$ & $0.6 * *$ \\
\hline
\end{tabular}

\begin{tabular}{|c|c|c|c|}
\hline Variables & Complications $(n=28)$ & No complications $(n=75)$ & p-value \\
\hline Mean age & $42.39 \pm 15.42$ & $40.24 \pm 14.89$ & $0.52 *$ \\
\hline Mean Serum Creatinine & $1.29 \pm 0.75$ & $1.49 \pm 1.43$ & $0.48^{*}$ \\
\hline Mean Stone size & $14.61 \pm 4.85$ & $12.85 \pm 4.51$ & $0.09 *$ \\
\hline Mean Operative time & $49.36 \pm 16.82$ & $38.63 \pm 14.31$ & $0.002 *$ \\
\hline $\begin{array}{l}\text { Gender } n(\%) \\
\text { Male } \\
\text { Female }\end{array}$ & $\begin{array}{c}3: 1 \\
21(75) \\
7(25)\end{array}$ & $\begin{array}{c}2: 1 \\
49(65.3) \\
26(34.7) \\
\end{array}$ & $0.35^{* *}$ \\
\hline $\begin{array}{l}\text { Side } n(\%) \\
\text { Right } \\
\text { Left }\end{array}$ & $\begin{array}{l}14(50) \\
14(50)\end{array}$ & $\begin{array}{l}33(44) \\
42(56) \\
\end{array}$ & $0.58 * *$ \\
\hline $\begin{array}{l}\text { Stone former } \mathrm{n}(\%) \\
\text { Primary } \\
\text { Recurrent }\end{array}$ & $\begin{array}{c}20(71.4) \\
8(28.6)\end{array}$ & $\begin{array}{l}41(54.7) \\
34(45.3)\end{array}$ & $0.124^{* *}$ \\
\hline JJ stenting $\mathrm{n}(\%)$ & $15(53.6)$ & $34(45.3)$ & $0.46 * *$ \\
\hline Comorbid conditions present n (\%) & $9(32.1)$ & $27(36)$ & $0.72 *$ \\
\hline
\end{tabular}

\section{METHODOLOGY}

After obtaining Institutional Ethical Review Committee approval (\# 4544-SUR-ERC-16), this observational study was conducted at the Urology section, Aga Khan University Hospital, Karachi from January 2010 to December 2018. All patients $\geq 18$ years of age, who underwent semi-rigid URS for single radio-opaque proximal ureteral calculi $10 \mathrm{~mm}$ or larger, confirmed on non-contrast enhanced computerised tomography (CT) abdomen, were included. Patients with a solitary kidney, congenital anomalies in the urinary tract, pregnancy, those who already had J stent or nephrostomy tube in place, or urosepsis, were excluded. Proximal ureter was defined as ureter extending from pelvi-ureteric junction (PUJ) to the upper border of sacro-iliac (SI) joint.

Semi-rigid URS was performed by consultant urologists in lithotomy position under general anesthesia using 6.4/8.0 Fr semi-rigid Karl Storz ${ }^{\circledast}$ ureteroscope, with the aid of fluoroscopy. Pneumatic lithoclast was used for stone fragmentation, using Swiss LithoClast ${ }^{\circledR}$ Master (EMS, Nyon, Switzerland). Any device, such as stone cone/basket etc, was not used to prevent stone migration. Stone clearance was assessed on plain X-ray abdomen done at 4 weeks postoperatively. Patients with no evidence of radio-opaque density were regarded as stone-free. Ancillary procedures performed like SWL; and secondary URS was recorded. Complications like stone migration, fever, and hematuria $>48$ hours were graded as per the modified Clavien-Dindo grading system (MCG).

Data was analysed using SPSS ${ }^{\circledR}$ version 21 . Quantitative variables were reported as mean and standard deviation, and compared using independent sample t-test. Qualitative variables were reported as frequencies and percentages, and compared using Chi-square test. The efficiency quotient (EQ) was calculated using standard formula: ${ }^{7}$

$\mathrm{EQ}=\mathrm{SFR}(\%) \times 100 /(100+$ retreatment rate $(\%)+$ ancillary procedures $(\%)$.

\section{RESULTS}

During the study duration, a total of 2,709 semi-rigid uretero- 
scopies were performed. Among those, 404 URS cases were done for proximal ureteral calculi. After applying the selection criteria, 103 cases were included in the final analysis. There were 70 males (68\%) and 33 females (32\%). Mean age was $40.83 \pm 14.92(18-76)$ years. Majority (66 \%) of patients presented in the Emergency Room and most common symptom presentation was flank or abdominal pain (77.7\%). Most patients (59.2\%) had the first episode of urolithiasis, while $40.8 \%$ were recurrent stone formers. Over $90 \%$ of the patients were of the American Society of Anesthesiology (ASA) grade 1 (53.4\%) and grade $2(40.8 \%)$. The mean serum creatinine was $1.44 \pm 1.28 \mathrm{mg} / \mathrm{dl}$, and the mean stone size was $13.33 \pm 4.64 \mathrm{~mm}(10-24 \mathrm{~mm})$.

All the patients admitted through the Emergency were discharged within 24 hours of the procedure. All elective procedures (34\%) were performed as day-care procedure. The mean surgery time was $41.50 \pm 15.60 \min$ (15-125 min). Failed access until the stone was reported in 12 $(11.7 \%)$ patients. Forty-nine $(47.6 \%)$ patients required indwelling J stent placement post-operatively, which were removed within 4 weeks. The reason for the placement of the stent was stone impaction, mucosal edema, and failure of negotiation of scope till stone.

At 4 weeks follow-up, the SFR was $83.5 \%$. Additional/ancillary procedures were performed in 17 (16.5\%) cases mainly SWL in 14 (13.5\%), and secondary URS in $3(2.9 \%)$ cases. Shock wave lithotripsy was done for migrated stone or stone fragments in 5 cases and for 9 patients with stones managed initially with the placement of J stent due to failed access. This ancillary procedure involved 1-2 sessions and was completed within 1-2 weeks of primary URS. Secondary URS was done within 2-3 weeks for 3 patients with initial failed access, who were later found on imaging to have a stone in either proximal or mid ureter. Our efficiency quotient (EQ) was 0.72 . Mean stone size was significantly larger $(15.91 \pm 5.53 \mathrm{~mm})$ in patients with failure of stone clearance compared to patients who were stone-free (12.82 $\pm 4.31 \mathrm{~mm}, \mathrm{p}=0.01$ ). None of the other clinical or demographic factors were significantly associated with stone clearance (Table I).

Overall complications were seen in 28 (27\%) patients and most common were Clavien grade I (21.5\%) including self-resolving hematuria for $>48$ hours (15.7\%) and fever (5.8\%). None of the patients required blood transfusion or bladder washout for hematuria. Stone migration was seen in $5(4.8 \%)$ patients, 3 with one or more fragments, and 2 with stone migration in toto. Only 1 patient developed grade IV complication, which was urosepsis. None of the patients had a ureteric injury, avulsion, or required conversion to an open procedure. Mean operative time was significantly longer $(49.36 \pm 16.82$ minutes) in patients with reported complications compared to patients without any complications (38.63 $\pm 14.31 \mathrm{~min}, \mathrm{p}<0.01$ ). No other demographic or clinical factor showed any significant association with complications
(Table II).

\section{DISCUSSION}

Proximal ureteral stones are among the most difficult ones to manage in urinary tract stone disease. ${ }^{8}$ Despite the advancement in the technology, larger proximal ureteral stones pose a challenge to minimally invasive treatment; and appropriate treatment option remains controversial. ${ }^{4}$

There are multiple options to manage proximal ureteral stones, i.e. conservative management, extracorporeal shock wave lithotripsy (SWL), semi-rigid URS, flexible URS, antegrade URS, PCNL or open ureterolithotomy with different success rate and complications. Choosing the best option for a particular patient is a major challenge for a treating physician. ${ }^{9}$

European Association of Urology (EAU) guidelines recommend ureteroscopic laser lithotripsy or SWL for proximal ureteral stones. ${ }^{10}$ Recent advances in the field of endourology have gradually shifted the balance in favour of flexible URS (with the laser) in developed countries. ${ }^{5}$

This approach, however, requires pre-procedure stenting and expertise for the handling of instruments. Moreover, these instruments are very expensive, fragile and require regular maintenance, which increases the cost enormously. In developing countries where cost and availability are the issues, cheaper alternatives with success and safety are preferred. ${ }^{6}$

Although SWL is a relatively cheap and minimally invasive outpatient procedure, it has disadvantage of long treatment time, higher re-treatment and failure rate, and is associated with poor patient compliance. ${ }^{4}$ PCNL and antegrade URS are invasive with the risk of bleeding and sepsis. Open and laparoscopic ureterolithotomy have very good stone clearance but at the cost of postoperative pain, longer procedure time, and prolonged hospital stay. ${ }^{9}$ Semi-rigid URS is a good alternative in developing countries ${ }^{6}$ but at a higher risk of access failure due to tight ureter and migration of stone to the kidney, which may require an additional procedure.

In a study from Egypt, Khairy-Salem et al. attempted to evaluate the outcome of semi-rigid URS for large proximal ureteral stones in 75 patients. ${ }^{6}$ Their study population had more females (55\%), while the present study population had mainly males (68\%). The mean stone size was $13.1 \mathrm{~mm}$, which is comparable to this study. They showed complete stone clearance in $60(80 \%)$ patients after primary URS with efficiency quotient (EQ) of 0.73 , comparable to this study result of 0.72 , but here the ancillary procedures rates were high $(16.3 \%)$ compared to $9.3 \%$ in their study.

Li et al. in a retrospective study found results similar to this study with semi-rigid URS achieving SFR of $86.5 \%$ at 4 weeks with overall complication rates of $23.3 \% .{ }^{11}$ Contrary to 
this study, which included larger proximal ureteral stones only, their study included stones of all sizes in the proximal ureter with a small number of cases with stones located in the renal pelvis as well.

Kumar et al. in a prospective RCT compared SWL and semi-rigid URS for proximal ureteral stones $<2 \mathrm{cms}$ in 90 patients. ${ }^{12}$ Their mean stone size was $12.5 \mathrm{~mm}$ in the URS group with 3 months SFR of $86.6 \%$ and the need for an ancillary procedure in $17.7 \%$. Their slightly higher stone-free rate could be due to use of holmium laser for stone fragmentation with potentially lesser chance of stone retropulsion as compared to pneumatic lithoclast, used in this study.

Sancak et al. found stone size, impaction, and ureteric stricture requiring dilatation as factors associated with failure of semi-rigid URS for proximal ureteral stone. ${ }^{13}$ Patients who did not achieve stone clearance in this study had significantly larger stone $(p<0.01) .{ }^{13}$ The correlation of stone impaction with failure of URS was not checked in this study.

Torricelli et al. performed a meta-analysis of randomised controlled trials (RCTs) comparing semi-rigid URS with laparoscopic ureterolithotomy (LU) for large proximal ureteral stones. ${ }^{14}$ They found semi-rigid URS to have shorter operative time $(p<0.001)$ and length of hospital stay $(p=0.03)$ compared to LU. In terms of efficacy, however, LU achieved a better overall SFR $(p<0.001)$ and lesser need for auxiliary procedures $(p<0.001)$.

Another meta-analysis compared URS with PCNL or LU for treatment of $>10 \mathrm{~mm}$ proximal ureteral stones. ${ }^{15}$ This study showed LU and PCNL to have a higher stone-free rate compared to URS but URS; was found to have shorter operative time, lesser hospital stay, and minimum complication rate. The authors recommended URS to be a standard treatment for larger proximal ureteral stones.

The overall complication rate in this study was $27.1 \%$ with majority Clavien grade 1 . In contrast to other studies, stone migration is categorised here as a complication (Clavien grade $3 b$ ) necessitating ancillary procedure. Only one patient developed urosepsis, which was managed conservatively. The complications rates are similar as reported in other studies. ${ }^{11-13}$

There are several limitations to this study, including retrospective study design, exclusion of radiolucent stones, and a limited record of perioperative MET. Long term follow-up of patients was not looked at.

The authors did not check the effect of hydronephrosis or stone impaction on stone clearance; neither did they perform stone analysis in all patients. Due to small sample size, they also did not perform regression analysis of the variables associated with stone clearance and complications. It was a single centre study, which investigated the efficacy and safety of semi-rigid URS for large proximal ureteral stones with no comparison group. The authors intend to compare the results of semi-rigid URS with other treatment modalities for large proximal ureteral stones in a prospective study in future.

\section{CONCLUSION}

Semi-rigid URS is an effective and safe procedure for the large proximal ureteral stones, with limited access to flexible instruments.

\section{DISCLOSURE:}

The study was presented as a moderated poster at the World Congress of Endourology 2019, at Abu Dhabi, UAE. Conference abstract was published in Journal of Endourology, Volume 33, Supplement 1, October 2019, Mary Ann Liebert, Inc. DOI: 10.1089/end.2019.29064.

\section{ETHICAL APPROVAL:}

This cohort study was carried out at AKUH after approval from University's Ethical Committee (ERC)-AKUH.

\section{PATIENTS' CONSENT:}

Since it was designed as a retrospective study, the data were collected from the hospital archive after approval of the Ethics Committee.

\section{CONFLICT OF INTEREST:}

The authors reported no conflict of interest.

\section{AUTHORS' CONTRIBUTION:}

MB: Conception and design, data acquisition and analysis, interpretation, drafting.

SMN: Conception and design, interpretation, drafting, critical revision, final approval.

S): Data acquisition and analysis, drafting.

MOA: Data acquisition and analysis, interpretation.

SN: Data acquisition and analysis, interpretation.

\section{REFERENCES}

1. Urkmez A, Tokuc E, Topaktas R, Sahin A, Yuksel OH. Mirabegron: A novel and promising medical expulsive treatment for ureteral stones? J Coll Physicians Surg Pak 2019; 29:73-4. doi: 10.29271/jcpsp.2019.01.73.

2. Ogreden E, Demirelli E, Aksu M, Tok DS, Oğuz U. Early ureteroscopic lithotripsy in acute renal colic caused by ureteral calculi. Int Urol Nephrol 2020; 52:15-19. doi: 10.1007/s11255-019-02298-9.

3. Conway JC, Friedman BW. Medical expulsive therapy (alpha blockers) for urologic stone disease. Acad Emerg Med 2020; doi: 10.1111/acem.13935.

4. Kadyan B, Sabale V, Mane D, Satav V, Mulay A, Thakur N, et al. Large proximal ureteral stones: Ideal treatment modality? Urol Ann 2016; 8:189-92. doi: 10.4103/09747796.157963.

5. Wang F, Yang $\mathrm{Y}$, Chen $\mathrm{H}$, Huang $\mathrm{H}$, Huang W, Weng $\mathrm{Z}$, Xie $\mathrm{H}$. The application of a single-use fiberoptic flexible ureteroscope for the management of upper urinary calculi. Int Urol 
Nephrol 2018; 50:1235-1241. doi: 10.1007/s11255-0181895-9.

6. Khairy-Salem $\mathrm{H}$, el-Ghoneimy $\mathrm{M}$, el-Atrebi M. Semirigid ureteroscopy in management of large proximal ureteral calculi: is there still a role in developing countries? Urology 2011; 77:1064-8. doi: 10.1016/j.urology.2010.08.067.

7. Ather MH, Nazim SM, Sulaiman MN. Efficacy of semirigid ureteroscopy with pneumatic lithotripsy for ureteral stone surface area of greater than $30 \mathrm{~mm} 2$. J Endourol 2009; 23:619-22. doi: 10.1089/end.2008.0182.

8. Ahmed AF, Maarouf A, Shalaby E, Alshahrani S, El-Feky M, Khaled $S$, et al. Semi-rigid ureteroscopy for proximal ureteral stones: Does adjunctive tamsulosin therapy increase the chance of success? Urol Int 2017; 98:411-417. doi: 10.1159/000452926.

9. Desai M, Sun Y, Buchholz N, Fuller A, Matsuda T, Matlaga B, et al. Treatment selection for urolithiasis: percutaneous nephrolithotomy, ureteroscopy, shock wave lithotripsy, and active monitoring. World J Urol 2017; 35:1395-1399. doi: 10.1007/s00345-017-2030-8.

10. Türk C, Petř́k A, Sarica K, Seitz C, Skolarikos A, Straub M, et al. EAU guidelines on diagnosis and conservative management of urolithiasis. Eur Urol 2016; 69:468-74. doi: 10.1016/j.eururo.2015.07.040.

11. Li K, Liao Z, Lin T, Li Z, He W, Liu C, et al. A novel semirigid ureterorenoscope with vacuum suctioning system for management of single proximal ureteral and renal pelvic stones: An initial experience. J Endourol 2018; 32(12): 1154-1159. doi: 10.1089/end.2018.0565.

12. Kumar A, Nanda B, Kumar N, Kumar R, Vasudeva P, Mohanty NK. A prospective randomised comparison between shockwave lithotripsy and semirigid ureteroscopy for upper ureteral stones <2 cm: A single centre experience. J Endourol 2015; 29(1):47-51. doi: 10.1089/end. 2012.0493.

13. Sancak EB, Kılınç MF, Yücebaş SC. Evaluation with decision trees of efficacy and safety of semirigid ureteroscopy in the treatment of proximal ureteral calculi. Urol Int 2017; 99:320-325. Urol Int 2017; 99(3):320-5. doi: 10.1159/ 000474954.

14. Torricelli FC, Monga M, Marchini GS, Srougi M, Nahas WC, Mazzucchi E. Semi-rigid ureteroscopic lithotripsy versus laparoscopic ureterolithotomy for large upper ureteral stones: A meta-analysis of randomized controlled trials. Int Braz J Urol 2016; 42(4):645-54. doi: 10.1590/S1677-5538.IBJU.2015.0696.

15. Wu T, Duan X, Chen S, Yang X, Tang T, Cui S. Ureteroscopic lithotripsy versus laparoscopic ureterolithotomy or percutaneous nephrolithotomy in the management of large proximal ureteral stones: A systematic review and meta-analysis. Urol Int 2017; 99(3):308-19. doi: 10.1159/ 000471773. 